\title{
Dexmedetomidine for the treatment of acute pain from complex regional pain syndrome
}

\author{
Younghoon Jeon ${ }^{1}$, and Jae Sik Jang ${ }^{2}$ \\ Department of Anesthesiology and Pain Medicine, ${ }^{1}$ Kyungpook National University School of Dentistry, ${ }^{2}$ Kyungpook National \\ University School of Medicine, Daegu, Korea
}

Complex regional pain syndrome (CRPS) may develop the following symptoms: fractures, limb trauma, or lesions of the peripheral or central nervous system [1]. CRPS has characteristics similar to neuropathic pain (intense burning pain, allodynia, and hyperalgesia) and is associated with local edema and symptoms suggestive of autonomic involvement (altered sweating, skin color, skin temperature in the affected limb). Pharmacotherapy is based on individual symptoms and it includes steroids, free radical scavengers, opioids, tricyclic antidepressants (TCA), sodium blocking agents, and N-methylD-aspartic acid (NMDA) receptor blocking agents. Although the pathophysiology of CRPS is not fully understood, it is suggested that sympathetically mediated pain is one of the mechanisms of CRPS [2].

Therefore, selective sympatholytic blocks (e.g., stellate ganglion block) for pain and symptom relief in CRPS patients are useful therapeutic options. Alpha-2 adrenergic receptor agonist has a centrally mediated reduction of the sympathetic nervous system activity [3]. It has been widely used as an analgesic adjuvant in perioperative conditions and pain therapy. Dexmedetomidine is a more selective $\alpha 2$-adrenergic receptor agonist with a shorter elimination half-life compared to clonidine. We successfully treated a 43-year-old female with acute pain from refractory CRPS type 1 using dexmedetomidine after therapy with ketamine, and lidocaine, and with a stellate ganglion block had failed. She complained of pallor, and presented with dystrophic skin with decreased temperature allodynia and hyperalgesia in the left wrist and hand. She took gabapentin $600 \mathrm{mg}$ and oxycontin $20 \mathrm{mg}$ daily. However, her pain intensity was 8 on a visual analogue scale of 10 (VAS: $0=$ "no pain" and $10=$ "worst pain imaginable"). We controlled her pain by continuous cervical epidural block with $0.2 \%$ ropivacaine. However, the epidural catheter was removed because of malposition of the epidural catheter, and then, her pain was treated with gabapentin 1,800 mg, oxycodone $40 \mathrm{mg}$, and amitriptyline $10 \mathrm{mg}$ daily. In addition, she received a stellate ganglion block with $1 \%$ lidocaine $7 \mathrm{ml}$ every other day. Her pain intensity decreased to $4-5$ on the VAS. However, about a month after these treatments, her pain intensity suddenly increased to 9 on the VAS. She complained of extremely sharp, bursting pain in her wrist and hand. After SGB with $1 \%$ lidocaine, the pain intensity decreased to $6-7$ on the VAS. However, about one hour after SGB, the patient complained of severe pain with a VAS score of 9 again. Therefore, we started ketamine therapy with a bolus infusion of $10 \mathrm{mg}$ followed by a continuous infusion dose of $10 \mu \mathrm{g} / \mathrm{kg} / \mathrm{hr}$. Twenty minutes after the infusion of the ketamine, the pain intensity decreased to a VAS score of $6-7$, but she complained of severe nausea and dizziness; therefore, we stopped the ketamine therapy. Furthermore, the infusion of lidocaine $200 \mathrm{mg}$ for 15 minutes did not resolve this situation. SGB with $1 \%$ lidocaine was effective in decreasing the pain, but its effect was limited due to the duration of lidocaine. We presumed that sympatholysis might be useful for relief of her symptoms. Dexmedetomidine reduces the sympathetic nervous system activity and is useful in diminishing catecholamine release through its sympatholytic properties [3]. Therefore, we infused dexmedetomidine at a loading dose of $0.5 \mu \mathrm{g} / \mathrm{kg}$ over 15 minutes and a continuous infusion dose of $0.2 \mu \mathrm{g} / \mathrm{kg} / \mathrm{hr}$ for 3

Corresponding author: Younghoon Jeon, M.D., Ph.D., Department of Anesthesiology and Pain Medicine, Kyungpook National University School of Dentistry, Samdeok-dong 2-ga, Jung-gu, Daegu 700-721, Korea. Tel: 82-53-420-5871, Fax: 82-53-426-2760, E-mail: jeon68@knu.ac.kr (c) This is an open-access article distributed under the terms of the Creative Commons Attribution Non-Commercial License (http:// creativecommons.org/licenses/by-nc/3.0/), which permits unrestricted non-commercial use, distribution, and reproduction in any medium, provided the original work is properly cited. 
hours. After infusion of dexmedetomidine, her pain decreased to a VAS score of 2 without any side effects such as bradycardia or hypotension. This effect persisted for three days. After three days, she complained of pain with a VAS score of 5-7 again. The patient received an infusion of dexmedetomidine again, and her VAS score decreased to $2-3$ for 4 days. One week later, she received a spinal cord stimulator, and then, her pain was maintained at a VAS score of $2-3$ at the three month follow up.

Central nervous system activation of postsynaptic $\alpha_{2 \mathrm{~A}}$ adrenergic receptors results in the inhibition of sympathetic activity [3]. Thus, alpha-2 adrenergic receptor agonist has the potential therapeutic properties to treat CRPS pain by preventing sympatho-afferent coupling and decreasing sympathetic outflow. In a previous report, a single shot of dexmedetomidine as adjuvant therapy for ketamine was effective in controlling acute pain from CRPS [4]. However, ketamine cannot be a feasible option because of the side effects such as blurred vision, drowsiness, nausea, and vomiting. In this case, the patient refused ketamine therapy because of severe nausea and drowsiness. However, infusion of dexmedetomidine alone was effective in controlling acute pain from CRPS.

Although the elimination half-life of dexmedetomidine is short ( $2-3$ hours), the analgesic-sparing effect of dexmedetomidine administered intraoperatively lasts up to 24 to 48 hours after surgery [5]. In this case, pain from CRPS significantly decreased for 3-4 days after the infusion of dexmedetomidine. The mechanism for the dexmedetomidine prolonged analgesic effect in this patient is not well understood and further research is needed.

In conclusion, dexmedetomidine may be an alternative therapeutic option for the treatment of acute pain from CRPS when other treatments fail. Further research is needed to define its role in managing chronic pain conditions like CRPS.

\section{References}

1. van Eijs F, Stanton-Hicks M, Van Zundert J, Faber CG, Lubenow TR, Mekhail N, et al. Evidence-based interventional pain medicine according to clinical diagnoses. 16. Complex regional pain syndrome. Pain Pract 2011; 11: 70-87.

2. Bruehl S. An update on the pathophysiology of complex regional pain syndrome. Anesthesiology 2010; 113: 713-25.

3. Coursin DB, Coursin DB, Maccioli GA. Dexmedetomidine. Curr Opin Crit Care 2001; 7: 221-6.

4. Nama S, Meenan DR, Fritz WT. The use of sub-anesthetic intravenous ketamine and adjuvant dexmedetomidine when treating acute pain from CRPS. Pain Physician 2010; 13: 365-8.

5. Gurbet A, Basagan-Mogol E, Turker G, Ugun F, Kaya FN, Ozcan B. Intraoperative infusion of dexmedetomidine reduces perioperative analgesic requirements. Can J Anaesth 2006; 53: 646-52. 